\title{
An Artificial Sequential Immune Responses Model for Anomaly Detection
}

\author{
Wen Zhou \\ Wuhan University \\ Wuhan, China \\ zw_mmwh@whu.edu.cn
}

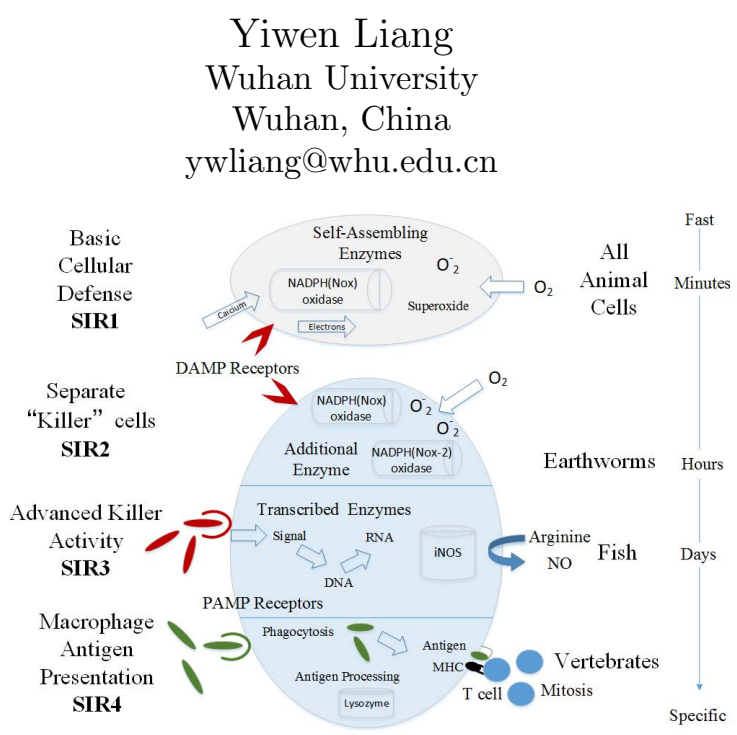

Figure 1: Human SIR mechanism[5]

\section{CCS CONCEPTS}

- Security and privacy $\rightarrow$ Artificial immune systems;

\section{KEYWORDS}

Sequential immune responses, Artificial immune system, Negative selection model, Danger theory, Anomaly Detection

\section{ACM Reference Format:}

Wen Zhou and Yiwen Liang. 2020. An Artificial Sequential Immune Responses Model for Anomaly Detection. In Proceedings of the Genetic and Evolutionary Computation Conference 2020 (GECCO '20 Companion). ACM, New York, NY, USA, 2 pages. https: //doi.org/10.1145/3377929.3389995

\section{THE HUMAN SIR MECHANISM}

Over the past years, a new immune mechanism has been proposed. It is called the SIR model, and its chief advocate is Charles D. Mills [5]. In Mills' paper, the classical negative selection model[2] and danger theory 4] are concentrate on how the immune responses are initiated. However, initiation is not protection, SIR describes the actual immune response that provides protection. It is derived from a comprehensive analysis of the immune system and confirms that a small number of disparate innate immune responses have different

Permission to make digital or hard copies of part or all of this work for personal or classroom use is granted without fee provided that copies are not made or distributed for profit or commercial advantage and that copies bear this notice and the full citation on the first page Copyrights for third-party components of this work must be honored. For all other uses, contact the owner/author(s).

GECCO '20 Companion, July 8-12, 2020, Cancún, Mexico

(C) 2020 Copyright held by the owner/author(s).

ACM ISBN 978-1-4503-7127-8/20/07.

https://doi.org/10.1145/3377929.3389995

response times and provide protection for the host. A key feature of SIR is that with the addition of new "protective layers", animals retain the evolutionarily older NADPH oxidase and NO-innately mediated protective responses. Moreover, different protection reactions that occur in vertebrates at very different rates (for example, from minutes to days). Nevertheless, the specific process of collaboration between different cells in SIR mechanism to elicit an immune response is not very clear. Therefore, the mechanism contains enough potentially interesting ideas to make it worth assessing its relevance to AIS.

Figure 1 depicts how we might picture an immune response based on SIR [5]. According to the immune response rate, a SIR model includes four layers: basic cellular defense (SIR1), separate killer cells (SIR2), advanced killer activity (SIR3) and macrophage antigen presentation (SIR4). In SIR1, the rapidly activated enzymes is presented to do immune response in all animal cells. SIR2 is mediated by the first immune cell: macrophage-like cells. SIR3 provides enhanced protection through advanced macrophage recognition and killing of pathogens, or other innate immune cells such as neutrophils. SIR4 can present antigens to T cells by DCs or macrophages, and then $\mathrm{T}$ kill cell can kill antigens, B lymphocytes can secrete antibodies for killing. As you can see, each layer of SIR model has its own unique advantages and disadvantages, therefore, the body is protected by multi-level cooperation. The SIR provides an overall framework for immune response.

Figure 2 shows a more detailed picture of how a SIR model can be viewed as an extension of immune signals. 


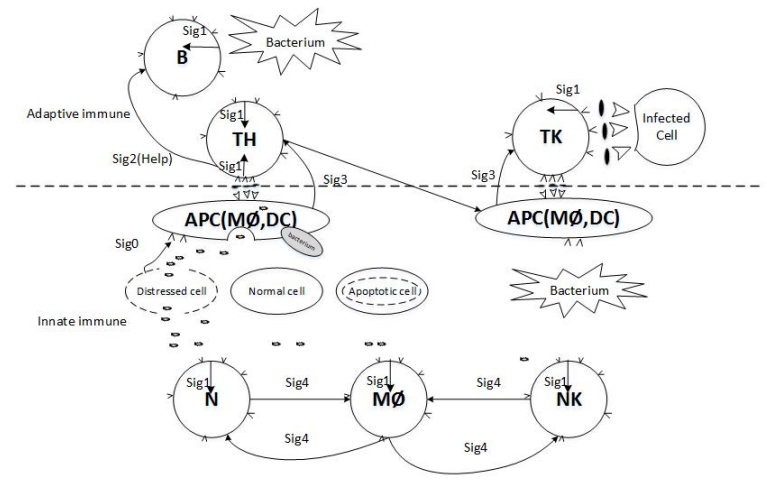

Figure 2: SIR mechanism

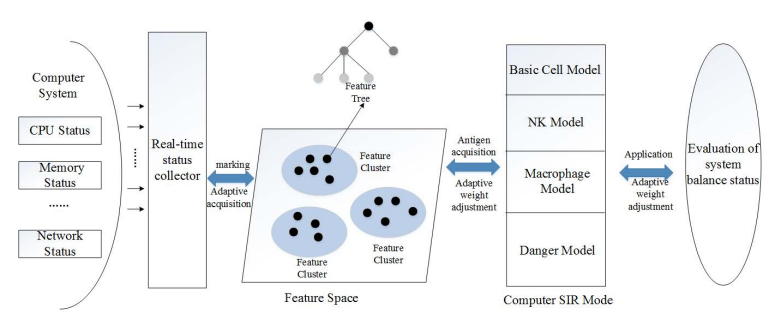

Figure 3: Artificial SIR model

The descriptions of the signals involved in Figure 2 are as follows: Sig0: Danger signal, Sig1: Antigen recognize signal, Sig2: T helper signal, Sig3: Co-stimulation signal, Sig4: Antiinflammatory and pro-inflammatory signals.

\section{THE ARTIFICIAL SIR MECHANISM AND ITS APPLICATIONS}

SIR mechanism is a complex biological model, and we have touched on only a few. It may be beneficial to list a number of considerations for an AIS practitioner regarding the suitability if artificial SIR mechanism for their application, such as the following considerations: (1)Artificial SIR mechanism is a coordination mechanism rather than a separate algorithm; (2)Artificial SIR model contains multiple immune cells;(3)In complex systems, macrophages have many abilities: kill, immunoregulatory properties and antigen presentation;(4)Each cell has complex immune response in its own area, while multiple cells cooperate with each other. The immune response area in biology is spatial. In the specific artificial immune application, distance calculation method is used to describe the immune response area; (5)The immune response is the labeling and killing of immune cells. Analogies to the artificial immune system, it can be used to label the anomaly behaviors, and decide to ignore, isolate or kill these anomaly behaviors according to the abnormality. In order to further illustrate the application of artificial SIR mechanism, this paper gives a preliminary and architectural diagram of its application in computer security environment assessment, as shown in Figure 3.
The hierarchical cooperative SIR is core of computer security situation assessment. There are many types of features in computer system and they have multi-source heterogeneity. For different features, the feature index space is established first, divided by cluster, and the corresponding feature tree is established. For different features, different models can be used for collaborative processing to find anomalies. Through targeted coordinated treatment of features and hierarchical feedback, potential danger can be detected and prevented quickly, providing a basis for the analysis and evaluation of computer operation status. By adopting the method of "taking the right medicine" and starting from the anomaly nature, it can effectively reduce the complexity of the problem and improve the self-adaptability of danger detection.

The point of artificial SIR mechanism is the central regulatory mechanism and anomaly processing. The central regulation mechanism can be regarded as an optimized function while dealing with data mining problems. Each immune cell serves as the main part of data mining, and meanwhile the function of exception handling can be ignored. As at present, many artificial immune algorithms based on biological immune mechanism are implemented in intrusion detection in cloud environment [1, intrusion detection in wireless sensor networks [6] and smart grid network attack detection [3, these applications are essentially a classification problem, therefore, considering the manual SIR mechanism, it can also be implemented in these fields.

\section{CONCLUSION AND FUTURE WORK}

This paper presents an artificial SIR mechanism, describes the functions of each level of the mechanism in details, and gives the field of application of its issues, but it is not limited to these areas. To continue this research, it is necessary to refine the artificial SIR model framework established in this paper, describe the cells and functions of each level of SIR in a formal way, and establish corresponding algorithms at each level, finally implemented in a specific field to verify the effectiveness of artificial SIR mechanism.

\section{REFERENCES}

[1] Azuan Ahmad, Norbik Bashah Idris, and Mohd Nazri Kama. 2017. CloudIDS: Cloud intrusion detection model inspired by dendritic cell mechanism. International Journal of Communication Networks and Information Security 9, 1 (2017), 67-75.

[2] Frank Macfarlane Burnet. 2010. Immunological Recognition of Self. Science 33, 1 (2010), 8.

[3] Obinna Igbe, Ihab Darwish, and Tarek Saadawi. 2017. Deterministic dendritic cell algorithm application to smart grid cyber-attack detection. In 2017 IEEE 4 th International Conference on Cyber Security and Cloud Computing (CSCloud). IEEE, 199-204.

[4] P Matzinger. 1994. Tolerance, Danger, and the Extended Family. annual review of immunology 12, 1 (1994), 991-1045.

[5] Charles D. Mills, Klaus Ley, Kurt Buchmann, and Johnathan Canton. 2015. Sequential Immune Responses: The Weapons of Immunity. Journal of Innate Immunity (2015), 444-450.

[6] Xin Xiao and Ruirui Zhang. 2017. Study of immune-based intrusion detection technology in wireless sensor networks. Arabian Journal for Science and Engineering 42, 8 (2017), 3159-3174. 